Pacific Journal of Mathematic 


\title{
REMARKS ON THE NEUMANN-POINCARE INTEGRAL EQUATION
}

\author{
Lars V. Ahlfors
}

1. Introduction. Neumann's method for solving the first and second boundary value problems lends itself readily to numerical computation. The degree of success depends primarily on the rate of convergence of the Neumann series, and the motivation for the present paper arose from a study of this question. The only new result is an estimate of the convergence rate by means of quasi-conformal mappings, which aside from its practical applications seems to present some theoretical interest. The rest of the paper is concerned with a generalization of the classical approach.

2. The Hilbert transform. Let $d \mu$ be a complex mass-distribution in the plane with compact carrier and total mass zero. The energy of the mass distribution is defined by

$$
\|d \mu\|^{2}=-\pi^{-1} \iint \log |t-\tau| d \mu(t) d \bar{\mu}(\tau) .
$$

With this norm the distributions with finite energy form a linear space which can be completed to a Hilbert space $\varepsilon$.

The Hilbert transform

$$
f(z)=\frac{1}{2 \pi i} \int \frac{d \mu(\zeta)}{\zeta-z}
$$

defines a function which is analytic outside of the carrier with a double zero at $\infty$. If the energy is finite one proves that $f(z)$ exists a.e. and

$$
2 \iint_{\Omega}|f|^{2} d x d y=\|d \mu\|^{2} \quad(z=x+i y),
$$

where $\Omega$ denotes the whole plane.

This result, to which there seems to be no convenient reference, is readily established by use of Fubini's the orem and the relation

Received September 15, 1951. The paper was written under Contract N5ori-07634 between Harvard University and the Office of Naval Research. It was initiated in connection with a program of numerical computations of conformal mappings directed by G. Birkhoff.

Pacific J. Math. 3 (1952), 271-280 


$$
\frac{1}{2 \pi} \underset{|z|<R}{\iint} \frac{d x d y}{(t-z)(\bar{\tau}-\bar{z})}=\log R-\log |t-\tau|+O\left(R^{-2}\right) .
$$

3. The inverse transformation. We regard (1) as a mapping $\phi$ which carries $d \mu$ into the differential

$$
f d z=\phi(d \mu)
$$

considered as element of a Hilbert space $r$ with the norm

$$
\|f d z\|^{2}=2 \iint_{\Omega}|f|^{2} d x d y .
$$

The inverse transformation $\phi^{-1}$ exists on a dense subspace of 7 . Indeed, let $\mathcal{F}^{\prime}$ consist of those $f d z$ for which $f$ is continuously differentiable and

$$
\frac{\partial f}{\partial \bar{z}}=\frac{\circ}{2}\left(\frac{\partial f}{\partial z}+i \frac{\partial f}{\partial y}\right)
$$

vanishes outside of a compact set. Now $\mathcal{F}^{\prime}$ is dense in $\mathcal{F}$, and for $f d z \in \mathcal{F}^{\prime}$ we can define a mass-distribution with density $-2 i \partial f / \partial \bar{z}$. Its Hilbert transform is

$$
-\frac{1}{\pi} \iint_{\Omega} \frac{\partial f / \partial \bar{\zeta} d \xi d \eta}{\zeta-z} \quad(\zeta=\xi+i \eta) .
$$

Integration by parts after removal of a small circle centered at $z$ shows that this integral has the value $f(z)$.

It follows that $\phi$ can be extended to an isometric transformation of $\varepsilon$ onto F.

If $C$ is a fixed compact set the differentials $f d z$ such that $f$ is analytic outside of $C$ form a closed subspace $\mathcal{F}_{0}$ of $\mathcal{F}^{\mathcal{F}}$. Its image under $\phi^{-1}$ is the closure $\varepsilon_{0}$ of the set of mass-distributions over $C$.

4. Anti-linear mappings. In $\varepsilon$ an involutory anti-linear mapping $J$ is defined by complex conjugation:

$$
J d \mu=d \bar{\mu} .
$$

A corresponding anti-linear mapping in $\mathcal{F}$ is defined by

$$
J^{\prime}=\phi J \phi^{-1} \text {. }
$$

For convenience we shall frequently write

$$
\begin{aligned}
& f d z=\phi(d \mu), \\
& g d z=\phi(d \bar{\mu}),
\end{aligned}
$$


where $g d z$ is thus the transform of $f d z$ under $J^{\prime}$. Clearly,

$$
\|f d z\|=\|g d z\| \text {. }
$$

We shall also consider

$$
d V=i(f d z-\bar{g} d \bar{z})
$$

and its conjugate differential

$$
d V^{*}=f d z+\bar{g} d \bar{z} .
$$

If $d \mu$ is real, then $f=g$, and $d V=-2 d f d z, d V^{*}=2 R f d z$.

5. Transformations in $\varepsilon_{0}$ and $\mathcal{F}_{0}$. Given a compact set $C$ we now restrict our attention to the subspaces $\varepsilon_{0}$ and $\mathcal{F}_{0}$ introduced at the end of $\S 2$. We assume further that $C$ has zero area, and that the complement of $C$ can be divided in two open sets $B_{i}$ and $B_{e}$, the interior and exterior of $C$.

In these circumstances (2) is more than a formal differential; in $B_{i}+B_{e}$ it is the differential of the logarithmic potential

$$
V(z)=-\pi^{-1} \int_{C} \log |\zeta-z| d \mu(\zeta) .
$$

Moreover, the Dirichlet integral $D(V)$, defined as

$$
D(V)=\iint_{\Omega}\left(\left|\frac{\partial V}{\partial x}\right|^{2}+\left|\frac{\partial V}{\partial y}\right|^{2}\right) d x d y,
$$

can be expressed by

$$
D(V)=\iint_{\Omega}\left(|f|^{2}+|g|^{2}\right) d x d y=\|f d z\|^{2}=\|d \mu\|^{2} .
$$

Let us now introduce the linear operator $\Lambda_{i}$ which transforms $f d z$ into $f_{i} d z$, where $f_{i}=f$ for $z \in B_{i}$ and $f_{i}=0$ for $z \in B_{e}$, and a similarly defined operator $\Lambda_{e}$. Then $\Lambda_{i}$ and $\Lambda_{e}$ are evidently projections on orthogonal subspaces, and

$$
\Lambda_{i}+\Lambda_{e}=I,
$$

where $I$ is the identity transformation.

Corresponding to $\Lambda_{i}$ and $\Lambda_{e}$ we define the operators

$$
\begin{aligned}
& L_{i}=\phi^{-1} \Lambda_{i} \phi, \\
& L_{e}=\phi^{-1} \Lambda_{e} \phi
\end{aligned}
$$

on $\varepsilon_{0}$. They are of course also projections, and $L_{i}+L_{e}$ is the identity trans- 
formation.

The fundamental role in the Neumann-Poincare theory is played by the transformation

$$
T=\frac{1}{2}\left[L_{i}-L_{e}+J\left(L_{i}-L_{e}\right) J\right]
$$

It operates in $\varepsilon_{0}$; the corresponding transformation in $\mathcal{F}_{0}$ is

$$
S=\frac{1}{2}\left[\Lambda_{i}-\Lambda_{e}+J^{\prime}\left(\Lambda_{i}-\Lambda_{e}\right) J^{\prime}\right]^{1}
$$

We note first that $J T J=T$; this means that $T$ is a real operator. Secondly, $J L_{i} J$ and $J L_{e} J$ are also projections. For this reason $T$ is self-adjoint with a norm $\leq 1$. We find explicitly

$$
(d \mu, T d \mu)=\frac{1}{2}\left[\left\|L_{i} d \mu\right\|^{2}+\left\|L_{i} d \bar{\mu}\right\|^{2}-\left\|L_{e} d \mu\right\|^{2}-\left\|L_{e} d \bar{\mu}\right\|^{2}\right]
$$

This can also be written in the form

$$
(d \mu, T d \mu)=\iint_{\Omega}\left(\left|f_{i}\right|^{2}+\left|g_{i}\right|^{2}-\left|f_{e}\right|^{2}-\left|g_{e}\right|^{2}\right) d x d y=D\left(V_{i}\right)-D\left(V_{e}\right) .
$$

Since

$$
\|d \mu\|^{2}=D\left(V_{i}\right)+D\left(V_{e}\right)
$$

we obtain

$$
|(d \mu, T d \mu)| \leq\|d \mu\|^{2},
$$

and this inequality implies

$$
\|T d \mu\| \leq\|d \mu\|
$$

As a matter of fact, the norm of $T$ is given by

$$
\|T\|=\sup \frac{\left|D\left(V_{i}\right)-D\left(V_{e}\right)\right|}{D\left(V_{i}\right)+D\left(V_{e}\right)}
$$

6. The Neumann problem. In the classical Neumann-Poincare theory, $C$ is a smooth curve and $d \mu$ a very regular mass-distribution on $C$. Under these condi-

${ }^{1}$ The operator $S J^{\prime}=J^{\prime} S$ is closely related to the operator $\mathbf{T}$ introduced by Schiffer and Bergman [1]. If $J^{\prime} S f d z=F d z$, the function $F$ is equal to the Schiffer-Bergman transform $\mathbf{T} f$ in $B_{i}$. 
tions the Hilbert transform

$$
f(z)=\frac{1}{2 \pi i} \int_{C} \frac{d \mu(\zeta)}{\zeta-z}
$$

satisfies the fundamental saltus-condition

$$
\left[f_{i}(\zeta)-f_{e}(\zeta)\right] d \zeta=d \mu(\zeta)
$$

where $f_{i}(\zeta)$ and $f_{e}(\zeta)$ are the interior and exterior boundary values on $C$.

For the differentials $d V$ and $d V^{*}$ the corresponding saltus-conditions are

$$
\begin{aligned}
& d V_{i}-d V_{e}=0, \\
& d V_{i}^{*}-d V_{e}^{*}=2 d \mu .
\end{aligned}
$$

To solve Neumann's problem for the interior means to solve an equation of the form

$$
d V_{i}^{*}=d y,
$$

where $d y$ is subject to the condition

$$
\int_{C} d y=0
$$

Because of (4) the equation (5) can also be written in the form

$$
d \mu+\frac{1}{2}\left(d V_{i}^{*}+d V_{e}^{*}\right)=d \gamma
$$

and the corresponding problem for the exterior would lead to the equation

$$
d \mu-\frac{1}{2}\left(d V_{i}^{*}+d V_{e}^{*}\right)=d \gamma .
$$

In the general case there is no way of defining $d V_{i}^{*}$ and $d V_{e}^{*}$ directly. However, $f_{i}(\zeta) d \zeta$ may be interpreted as the saltus of $f_{i} d z$ where, as before, $f_{i}=f$ in the interior and $f_{i}=0$ in the exterior. For this reason, we can represent $f_{i}(\zeta) d \zeta$ by $\phi^{-1}\left(f_{i} d z\right)$, and a similar representation is obtained for $f_{e}(\zeta) d \zeta$. With these interpretations, we find that $(1 / 2)\left(d V_{i}^{*}+d V_{e}^{*}\right)$ is represented by $T d \mu$, and the equations (6), (7) obtain the form

$$
d \mu \pm T d \mu=d \gamma .
$$

In this sense the Neumann problem has a meaning under extremely general circumstances. 
Equation (8) is solved by Neumann's series

$$
d \mu=d \gamma \mp T d \gamma+T^{2} d \gamma \mp \cdots,
$$

provided that the series converges. Here, convergence is interpreted in terms of the energy-norm, and the necessary and sufficient condition for convergence is that the norm of $T$ is $<1$. More precisely, if $\|T d \mu\| \leq k\|d \mu\|$ the series has a geometric majorant with the ratio $k$.

It is thus of practical and theoretical importance to find upper bounds for the norm of $T$.

7. Quasi-conformality. We shall pursue the study of the convergence only for the case that $C$ is a Jordan curve, $B_{i}$ and $B_{e}$ being then simply connected on the sphere ${ }^{2}$. It may be expected that the norm of $T$ depends on the regularity and near-circularity of $C$. However, instead of introducing explicit regularity conditions, we shall make use of an implicit condition which seems to influence the norm much more directly.

Our only assumption will be that there exists a quasi-conformal mapping $\zeta(z)$ of $B_{i}$ onto $B_{e}$ which leaves $C$ fixed. More exactly, $\zeta(z)$ is supposed to be a topological mapping of $B_{i}+C$ onto $B_{e}+C$ such that $\zeta(z)=z$ on $C$. In $B_{i}$ we assume $\zeta(z)$ to be continuously differentiable, and we write

$$
\begin{aligned}
& p=\frac{\partial \zeta}{\partial z}=\frac{1}{2}\left(\frac{\partial \zeta}{\partial x}-i \frac{\partial \zeta}{\partial y}\right) \\
& q=\frac{\partial \zeta}{\partial \bar{z}}=\frac{1}{2}\left(\frac{\partial \zeta}{\partial x}+i \frac{\partial \zeta}{\partial y}\right)
\end{aligned}
$$

The Jacobian of the mapping is $|p|^{2}-|q|^{2}$; in the present case the mapping is sense-reversing, and hence $|p| \leq|q|$. The condition of quasi-conformality consists in requiring that

$$
|p| \leq k|q|
$$

for some $k<1$. The mapping is then said to have a maximal eccentricity $\leq k$ or a maximal dilatation $\leq(1+k)(1-k)$.

It can be shown that the existence of a quasi-conformal mapping is compatible with corners but excludes cusps.

${ }^{2}$ If $B_{i}$ is a multiply connected region, the harmonic measures of the contours correspond to solutions of $d \mu=T d \mu$. In this case the norm is thus exactly one. However, a significant problem arises if we consider only the sub-space for which the total mass vanishes separately on each contour. An even more natural approach is to embed $B_{i}$ in a surface of higher genus so that $B_{e}$ becomes connected [4]. 
8. Theorem. Our result can now be expressed as follows:

THEOREM. If $B_{i}$ and $B_{e}$ are complementary simply connected regions bounded by a Jordan curve $C$, and there exists a quas $i$-conformal mapping of $B_{i}$ onto $B_{e}$ which leaves $C$ fixed and whose maximal eccentricity is $\leq k$, then the norm of the transformation $T$ is at most $k$.

To make the essential steps of the proof clear, let $V$ be the potential of a distribution $d \mu$. The restrictions of $V$ to $B_{i}$ and $B_{e}$ being denoted by $V_{i}$ and $V_{e}$, as above, we compare $V_{i}(z)$ with $V_{e}(\zeta(z))$. These functions are equal on $C$, and hence.

$$
D\left(V_{i}(z)\right) \leq D\left(V_{e}(\zeta(z))\right)
$$

by Dirichlet's principle. But

$$
\begin{aligned}
& D\left(V_{e}(\zeta(z))\right)=2 \iint_{B_{i}}\left(\left|\frac{\partial V_{e}}{\partial z}\right|^{2}+\left|\frac{\partial V_{e}}{\partial \bar{z}}\right|^{2}\right) d x d y \\
& \quad=2 \iint_{B_{i}}\left(\left|\frac{\partial V_{e}}{\partial \zeta} p+\frac{\partial V_{e}}{\partial \bar{\zeta}} \bar{q}\right|^{2}+\left|\frac{\partial V_{e}}{\partial \zeta} q+\frac{\partial V_{e}}{\partial \bar{\zeta}} \bar{p}\right|^{2}\right) d x d y \\
& \leq 2 \iint_{B_{i}}\left(\left|\frac{\partial V_{e}}{\partial \zeta}\right|^{2}+\left|\frac{\partial V_{e}}{\partial \bar{\zeta}}\right|^{2}\right)(|p|+|q|)^{2} d x d y \\
& \quad=2 \iint_{B_{i}}\left(\left|\frac{\partial V_{e}}{\partial \zeta}\right|^{2}+\left|\frac{\partial V_{e}}{\partial \bar{\zeta}}\right|^{2}\right) \frac{|p|+|q|}{|q|-|p|} d \xi d \eta \leq \frac{1+k}{1-k} D\left(V_{e}\right),
\end{aligned}
$$

and we have proved that

$$
D\left(V_{i}\right) \leq \frac{1+k}{1-k} D\left(V_{e}\right)
$$

Since the inverse mapping of $\zeta(z)$ is also quasi-conformal with the same maximal dilatation, we obtain similarly

$$
D\left(V_{e}\right) \leq \frac{1+k}{1-k} D\left(V_{i}\right) .
$$

These inequalities imply

$$
\left|D\left(V_{i}\right)-D\left(V_{e}\right)\right| \leq k\left(D\left(V_{i}\right)+D\left(V_{e}\right)\right),
$$

and it follows by (3) that the norm of $T$ is $\leq k$.

In this proof the use of Dirichlet's principle needs justification. In other 
words, we must prove that the mixed Dirichlet integral

$$
D\left(V_{i}(z)-V_{e}(\zeta(z)), V_{i}(z)\right)
$$

vanishes. For this purpose it is sufficient to know that the potential $V(z)$ is continuous on $C$. Then $V_{i}(z)-V_{e}(\zeta(z))$ is continuously zero on the boundary. Moreover, we have shown that $V_{e}(\zeta(z))$ has a finite Dirichlet integral. Under these conditions the mixed Dirichlet integral vanishes according to a familiar theorem.

We have thus to show that $V(z)$ is continuous for a dense set of distributions in $\varepsilon_{0}$. Suppose that $w=\phi(z)$ defines a conformal mapping of $B_{e}$ onto $|w| \geq 1$. Set

$$
\begin{aligned}
& w=\phi(z) \text { for } z \in B_{e}, \\
& w=1 / \bar{\phi}(\zeta(z)) \text { for } z \in B_{i} .
\end{aligned}
$$

This is a quasi-conformal mapping of the whole $z$-plane onto the $w$-plane except for $C$ where the mapping is only known to be continuous. It is an important theorem in the theory of quasi-conformal mappings that such a mapping satisfies a Lipschitz condition of order $(1-k) /(1+k)$ on any compact set ${ }^{3}$. We have thus

$$
\begin{aligned}
& \left|\phi\left(z_{1}\right)-\phi\left(z_{2}\right)\right|<M\left|z_{1}-z_{2}\right|^{(1-k) /(1+k)} \\
& \left|z_{1}-z_{2}\right|<M\left|\phi\left(z_{1}\right)-\phi\left(z_{2}\right)\right|^{(1-k) /(1+k)}
\end{aligned}
$$

in a neighborhood of $C$.

From this behavior we draw two conclusions: 1) if a mass-distribution $d \mu$ of finite energy is carried from the $z$-plane to the $w$-plane by means of the mapping (10), then the energy remains finite, and vice versa; 2) if the potential is continuous in one plane, then it is also continuous in the other.

In the $w$-plane the distributions with continuous density in $|w|=1$ are dense and have a continuous potential. The corresponding distributions in the $z$-plane have the same property. This completes the proof.

9. An application. As an application, we consider the case of a star-shaped region $B_{i}$ whose boundary has the equation $r=r(\phi)$ in polar coordinates. A

${ }^{3}$ Assume that $\zeta(z)$ maps $|z|<1$ quasi-conformally onto $|\zeta|<1$ with dilatation $\leq K=$ $(1+k) /(1-k)$. The mapping can be extended to the double discs with branch-points at $z_{1}, z_{2}$ and $\zeta\left(z_{1}\right), \zeta\left(z_{2}\right)$. Conformally speaking the double discs can be regarded as annuli, and the existence of the mapping implies that the ratio of their modules must be $\leq K$. As $z_{1}$ and $z_{2}$ approach each other the modules are asymptotic to $-\log \left|z_{1}-z_{2}\right|$ and $-\log \left|\zeta\left(z_{1}\right)-\zeta\left(z_{2}\right)\right|$, and the Lipschitz condition follows. The proof is inspired by reasonings of Teichmüller. 
quasi-conformal mapping $\zeta(z)$ is obtained as a transformation by reciprocal radii:

$$
\zeta(z)=\frac{r(\phi)^{2}}{\bar{z}} .
$$

Since

$$
\frac{\partial \phi}{\partial z}=\frac{1}{2 i z}, \frac{\partial \phi}{\partial \bar{z}}=-\frac{1}{2 i \bar{z}},
$$

we obtain

$$
\begin{aligned}
& p=\frac{\partial \zeta}{\partial z}=-i \frac{r r^{\prime}}{|z|^{2}}, \\
& q=\frac{\partial \zeta}{\partial \bar{z}}=-\frac{r^{2}}{\bar{z}^{2}}+i \frac{r r^{\prime}}{\bar{z}^{2}} .
\end{aligned}
$$

Hence

$$
\frac{|p|}{|q|}=\frac{\left|r^{\prime}\right|}{\sqrt{r^{2}+r^{\prime 2}}}=\cos \theta,
$$

where $\theta$ is the angle between the tangent and the radius vector. If $\theta_{0}=\min \theta$ the Neumann series has thus a convergence ratio $\leq \cos \theta_{0}$.

10. Rate of convergence. For practical purposes it may not be sufficient to know that the series (9) converges in the energy norm. If $C$ is rectifiable we consider the Banach space $B$ of distributions $d \mu$ with continuous density on $C$ (and total mass zero) and use the maximum of the density as norm. Under sufficiently strong regularity conditions it is proved in the classical theory that $T$ maps $B$ upon itself, and even that $T$ is completely continuous on $B$.

If this is so, $T$ has a pure point-spectrum in $B$. But it is clear that every eigen-value in $B$ belongs to the spectrum with respect to $E_{0}$. In $E_{0}$ the absolute value $\left|\lambda_{0}\right|$ of the spectral point nearest to the origin is reciprocal to the norm. The smallest eigen-value in $B$ is $\geq\left|\lambda_{0}\right|$, and it follows that the Neumann series

$$
d y+\lambda T d y+\lambda^{2} T^{2} d y+\cdots
$$

converges in the B-norm for $|\lambda|<\left|\lambda_{0}\right|$. Therefore the convergence rate of

$$
d y+T d y+T^{2} d y+\cdots
$$

is comparable to that of a geometric series with ratio $\left|\lambda_{0}\right|^{-1}$.

An upper bound for the norm of $T$ in $\varepsilon_{0}$ can therefore serve as a practical estiof the rate of convergence in solving a Neumann problem by the iterative method. 


\section{References}

1. S. Bergmann and M. Schiffer, Kernel functions and conformal mapping, Compositio Math. 8 (1951), 205-249.

2. G. Birkhoff, D. M. Young, and E. H. Zarantonello, Numerical methods in conformal mapping, to appear in Proc. Symposia Appl. Math. 4.

3. S. A. Gerschgorin, Über die konforme Abbildung eines einfach zusammenhängenden Bereiches auf den Kreis, Mat. Sbornik 40 (1933), 48-58.

4. H. L. Royden, A modification of the Neumann-Poincaré method for multiply connected regions, Pacific J. Math. 2 (1952), 385-394.

Harvard UNiversity 


\title{
PACIFIC JOURNAL OF MATHEMATICS
}

\section{EDITORS}

\section{R. M. RobINSON \\ University of California \\ Berkeley 4, California}

\author{
*R. P. Dilwor TH \\ California Institute of Technology \\ Pasadena 4, California
}

\author{
E. F. BECKENBACH, Managing Editor \\ University of California \\ Los Angeles 24, California
}

${ }^{*}$ During the absence of Herbert Busemann in 1952.

\section{ASSOCIATE EDITORS}

$\begin{array}{llll}\text { R. P. DILWORTH } & \text { P. R. HALMOS } & \text { B } \emptyset \text { RGE JESSEN } & \text { J. J. STOKER } \\ \text { HERBERT FEDERER } & \text { HEINZ HOPF } & \text { PAUL LÉVY } & \text { E. G. STRAUS } \\ \text { MARSHALL HALL } & \text { R. D. JAMES } & \text { GEORGE PÓLYA } & \text { KÖSAKU YOSIDA }\end{array}$

\section{SPONSORS}

UNIVERSITY OF BRITISH COLUMBIA

CALIFORNIA INSTITUTE OF TECHNOLOGY

U NIVERSITY OF CALIFORNIA, BERKELEY

UNIVERSITY OF CALIFORNIA, DAVIS

UNIVERSITY OF CALIFORNIA, LOS ANGELES

UNIVERSITY OF CALIFORNIA, SANTA BARBARA

OREGON STATE COLLEGE

UNIVERSITY OF OREGON

\author{
UNIVERSITY OF SOUTHERN CALIFORNIA \\ STANFORD UNIVERSITY \\ WASHINGTON STATE COLLEGE \\ UNIVERSITY OF WASHINGTON \\ AMERICAN MATHEMATICAL SOCIETY \\ NATIONAL BUREAU OF STANDARDS, \\ INSTITUTE FOR NUMERICAL ANALYSIS
}

Mathematical papers intended for publication in the Pacific Journal of Miathematics should be typewritten (double spaced), and the author should keep a complete copy. Manuscripts may be sent to any of the editors. All other communications to the editors should be addressed to the managing editor, E. F. Beckenbach, at the address given above.

Authors are entitled to receive 100 free reprints of their published papers and may obtain additional copies at cost.

The Pacific Journal of Mathematics is published quarterly, in March, June, September, and December, by the University of California, Berkeley 4, California. The price per volume (4 numbers) is $\$ 8.00$; single issues, $\$ 2.50$. Special price to individual faculty members of supporting institutions and to individual members of the American Mathematical Society: $\$ 4.00$ per volume; single issues, $\$ 1.25$.

Subscriptions, orders for back numbers, and changes of address should be sent to the publishers, University of California Press, Berkeley 4, California.

Printed at Ann Arbor, Michigan. Entered as second class matter at the Post Office, Berkeley, California.

\section{UNIVERSITY OF CALIFORNIA PRESS • BERKELEY AND LOS ANGELES}




\section{Pacific Journal of Mathematics}

\section{Vol. 2, No. $3 \quad$ March, 1952}

Lars V. Ahlfors, Remarks on the Neumann-Poincaré integral equation .... 271

Leonard P. Burton, Oscillation theorems for the solutions of linear, nonhomogeneous, second-order differential systems ............ 281

Paul Civin, Multiplicative closure and the Walsh functions . . . . . . . . . . . 291

James Michael Gardner Fell and Alfred Tarski, On algebras whose factor algebras are Boolean .................................. 297

Paul Joseph Kelly and Lowell J. Paige, Symmetric perpendicularity in Hilbert geometries .................................. 319

G. Kurepa, On a characteristic property of finite sets .............. 323

Joseph Lehner, A diophantine property of the Fuchsian groups ......... 327

Donald Alan Norton, Groups of orthogonal row-latin squares ........... 335

R. S. Phillips, On the generation of semigroups of linear operators ....... 343

G. Piranian, Uniformly accessible Jordan curves through large sets of relative harmonic measure zero ........................ 371

C. T. Rajagopal, Note on some Tauberian theorems of $O . S z \tilde{A} ; s z \ldots \ldots \ldots 377$

Halsey Lawrence Royden, Jr., A modification of the Neumann-Poincaré method for multiply connected regions .................... 385

George H. Seifert, A third order irregular boundary value problem and the associated series ...................................... 395

Herbert E. Vaughan, Well-ordered subsets and maximal members of ordered sets....

Hans F. Weinberger, An optimum problem in the Weinstein method for eigenvalues.

Shigeki Yano, Note on Fourier analysis. XXXI. Cesàro summability of Fourier series. 